\title{
Transfusion demand control strategies: Potential efficacy of hospital and regional interventions
}

\author{
Philip J. Crispin ${ }^{\mathrm{a}, \mathrm{b}, *}$, Maria Burgess ${ }^{\mathrm{c}}$, Therese M. Crispin ${ }^{\mathrm{d}}$ \\ ${ }^{a}$ Haematology Department, Canberra Hospital, Garran ACT, Australia \\ ${ }^{\mathrm{b}}$ Australian National University Medical School, Acton ACT, Australia \\ ${ }^{\mathrm{c}}$ Department of the Chief Health Officer, ACT Health, Canberra, Australia \\ ${ }^{\mathrm{d}}$ Intensive Care Unit, Canberra Hospital, Garran ACT, Australia
}

\section{A R T I C L E I N F O}

\section{Keywords:}

Blood transfusion

Contingency plan

Blood shortage

\begin{abstract}
A B S T R A C T
Introduction: Contingency plans have been developed to direct appropriate responses to blood shortages. Planning requires an understanding of the potential savings of different conservation strategies.

Method: The Australian Capital Territory (ACT) Haemovigilance and ACT Pathology transfusion databases were reviewed from March to September 2003. All transfusion episodes were prioritised in accordance with the Australian National Blood Supply Contingency Plan. The number of red cell transfusions related to various indications, their appropriateness and acuity was determined. The potential reduction in red cell usage was modelled for potential red cell reduction interventions.

Results: There were 2305 units of red cells captured during the timeframes of the audits. This accounted for an estimated $70 \%$ of all red cell transfusions in the ACT. After correcting for the number of red cells transfused at each hospital, red cells were prioritised as category 1 in $59 \%, 2$ in $27 \%$ and 3 in 13\%. The remainder had insufficient data for classification. Transfusion for elective surgery accounted for $14.7 \%$ of red cells used, with $9.0 \%$ rated category 3 under the contingency plan.

There were $17.3 \%$ of red cells transfused for inappropriate indications, when reviewed against national guidelines. After excluding inappropriate transfusions, cancelling elective surgery could potentially save a further $5.5 \%$ and $4.3 \%$ of blood utilisation for category 3 and 2 patients, respectively. Significant differences were found between hospitals.

Conclusion: Targeting inappropriate transfusions by vetting particularly for inappropriate transfusions not only re-directs blood away from those unlikely to benefit, but is also more effective at preserving the red blood cells than other measures during times of supply limitation. Contingency planning needs to accommodate the variable case-mix in hospitals, allocate resources for transfusion medicine specialists to review every transfusion request and may be better coordinated at a jurisdictional level.
\end{abstract}

Crown Copyright @ 2010 Published by Elsevier Ltd. All rights reserved.

\section{Introduction}

Maintaining an adequate blood supply inventory requires a balance between supply and demand, but

\footnotetext{
* Corresponding author at: Canberra Hospital, P.O. Box 11, Woden ACT 2606, Australia. Tel.: +61 26244 3964; fax: +61 262442892.

E-mail address: Philip.crispin@act.gov.au (P.J. Crispin).
}

increased demand or reduced supply may lead to blood shortages. Supply limitation commonly occurs due to the impact of seasonal infections on the donor pool and typically impacts national blood reserves [1-3]. Pandemic infection has the potential to impact the blood supply in a very similar way, but may be more severe if there is a high incidence of infection [4]. Supply failure may also occur suddenly due to interruptions to the supply chain, 
particularly failure of key facilities or processes. Demand surges may occur due to natural or man-made disasters, which may also potentially impact on the ability to increase supply from local donors or re-stock from external blood banks.

Contingency plans have been developed to direct appropriate responses to blood shortages [5]. In Australia, the National Blood Supply Contingency Plan (NBSCP) was released in 2008 to guide the responses of governments, the blood bank, regulators, transfusion laboratories and hospitals [6]. The plan guides, rather than directs, strategies to be taken locally, but requires the development of jurisdictional and local plans to manage demand. Local plans should aim to ensure efficient inventory management, collaboration and communication and reductions in blood use proportionate to the expected shortfall in blood supply. A variety of strategies may be used to limit the demand for blood products. The aim of this study was to determine the potential for various strategies to restrict red blood cell utilisation within hospitals and local regions.

\section{Method}

Transfusion episodes were extracted from the ACT Haemovigilance databases. These were audits of consecutive transfusions conducted between March and September 2003 as a snapshot of transfusion practice in the Canberra region and have been previously described [7]. Canberra has an urban population of approximately 340,000 and services a geographically diverse regional referral area with an additional population of more than 200,000. There two public hospital and three private hospitals undertaking transfusions. Both public hospitals have emergency departments. One is the regional tertiary centre. The audits were conducted at one private and two public hospitals providing obstetric, trauma, oncology, haematology (including autologous stem cell transplant) and all major medical and surgical specialities, with the exception of solid organ transplantation.

All transfusion episodes were reviewed during or shortly after the relevant admission. The audit extracted data from the clinical record, clinicians and occasionally directly from patients and included the indications for and responses to transfusion, major procedures, diagnoses, co-morbidities, laboratory values pre and post transfusion and clinical responses where available. Transfusion episodes were defined by a single decision to transfuse red cells, irrespective of the number of red cell units prescribed. Appropriateness of transfusion was determined in accordance with published criteria [8].

All transfusion episodes were prioritised on the basis of the urgency of clinical need in accordance with the Australian NBSCP [6] by two investigators, and discrepancies resolved by consensus. Elective medical or surgical procedures are category 3 , indications which can be safely deferred for only a limited time, but which may carry some risk of increasing morbidity are category 2 , and urgent indications are category 1 . The definitions of each categorisation are summarised in Table 1. Massive transfusions and neonatal transfusions were excluded from the initial audit and identified from the pathology database. The number of red cell units potentially saved by various demand control strategies was then determined. The audit was carried out in accordance with the Declaration of Helsinki as well as National Health and Medical Research Council (NHMRC) [9] and ACT Health Human Research Ethics Committee requirements for the conduct of clinical audit.

\section{Results}

\subsection{Transfusions included}

The Haemovigliance database contained transfusions from two public and one private hospitals. These institu-

Table 1

Blood access priorities.

\begin{tabular}{|c|c|}
\hline Priority level & Suggested transfusion indications \\
\hline Blood Access & - Resuscitation \\
\hline \multirow[t]{7}{*}{ Priority 1} & $\begin{array}{l}\text { Life threatening or ongoing blood loss from any cause } \\
\text { - Surgical Support }\end{array}$ \\
\hline & $\begin{array}{l}\text { Emergency (death expected within } 24 \mathrm{~h} \text { without intervention) or urgent (patient likely to have major morbidity with } \\
\text { intervention) surgery }\end{array}$ \\
\hline & Non-deferrable organ transplantation \\
\hline & - Non surgical anaemia \\
\hline & Life threatening anaemia (including in utero) \\
\hline & Support for stem cell transplantation or chemotherapy that cannot be delayed \\
\hline & Severe bone marrow failure, haemoglobinopathies or other conditions where patients cannot tolerate delay in transfusion \\
\hline Blood Access & - Surgery \\
\hline \multirow[t]{4}{*}{ Priority 2} & Semi-urgent surgery (patient likely to have minor morbidity if not carried out) \\
\hline & Cancer surgery that cannot be deferred without risk \\
\hline & Symptomatic, non-life-threatening post operative or post partum anaemia \\
\hline & $\begin{array}{l}\text { - Nonsurgical anaemia } \\
\text { Symptomatic, non-life-threatening anaemia that cannot be managed by other means }\end{array}$ \\
\hline Blood Access & - Surgery \\
\hline Priority 3 & $\begin{array}{l}\text { Elective surgery requiring cross-matched red blood cell support of two or more units } \\
\text { - Nonsurgical anaemia } \\
\text { Other non-urgent medical indications for transfusion }\end{array}$ \\
\hline
\end{tabular}

* Adapted from [6]. 
tions are supplied with approximately $84 \%$ of ACT red cell units. The audit in the tertiary institution captured 544 transfusion episodes involving 1199 red cell units over an 82 day period, with a further 297 red cell units identified as having been excluded from the initial audit due to massive or neonatal transfusions. These episodes included approximately $83 \%$ of transfused units during this period. In the smaller hospitals 809 units were transfused during a 180 day audit period, in 360 episodes. These accounted for an estimated $96 \%$ of units during this timeframe. The audit periods were of differing durations to ensure an adequate number of red cell transfusions in each institution and major departments within each institution. The results from each institution were weighted to account for the difference in the audit durations.

There were significant differences between the institutions, likely reflecting the different functions and case mix of the hospitals. There were a greater number of transfusions to males at the tertiary centre, with a male:female ratio of 1.55 . This trend was reversed at the other hospitals where 0.77 males were transfused for every female $(p<0.05)$. The mean age of transfusion recipients was 61.7 years at the tertiary centre compared with 66.9 years at the other hospitals $(p<0.05)$.

\subsection{Blood utilisation}

The majority of transfusions (59\%) were for category 1 indications, $27 \%$ were for category 2 and $13 \%$ were category 3 , as defined in the NBSCP. Elective surgery accounted for $14.7 \%$ of transfused units and $9 \%$ of units were given during admissions for category 3 elective surgical admissions. The haematology, orthopaedic and gastroenterology departments used more red cells than other departments, as shown in Fig. 1. There was no association between the contingency plan priority levels and particular departments. Massive transfusions accounted for $16 \%$ of blood use, to $3.2 \%$ of patients, and most were not readily avoidable.

\subsection{Inappropriate transfusions}

Inappropriate transfusions, as defined by NHMRC criteria, accounted for at least $17.3 \%$ of red cells. Therefore preventing inappropriate transfusions offered the single largest potential for red cell reduction. Once inappropriate transfusions were excluded, category 3 elective surgery accounted for a further 5.5\% of transfusions and category 2 indications $4.2 \%$.

- Cardiothoracic

Surgery

$6 \%$

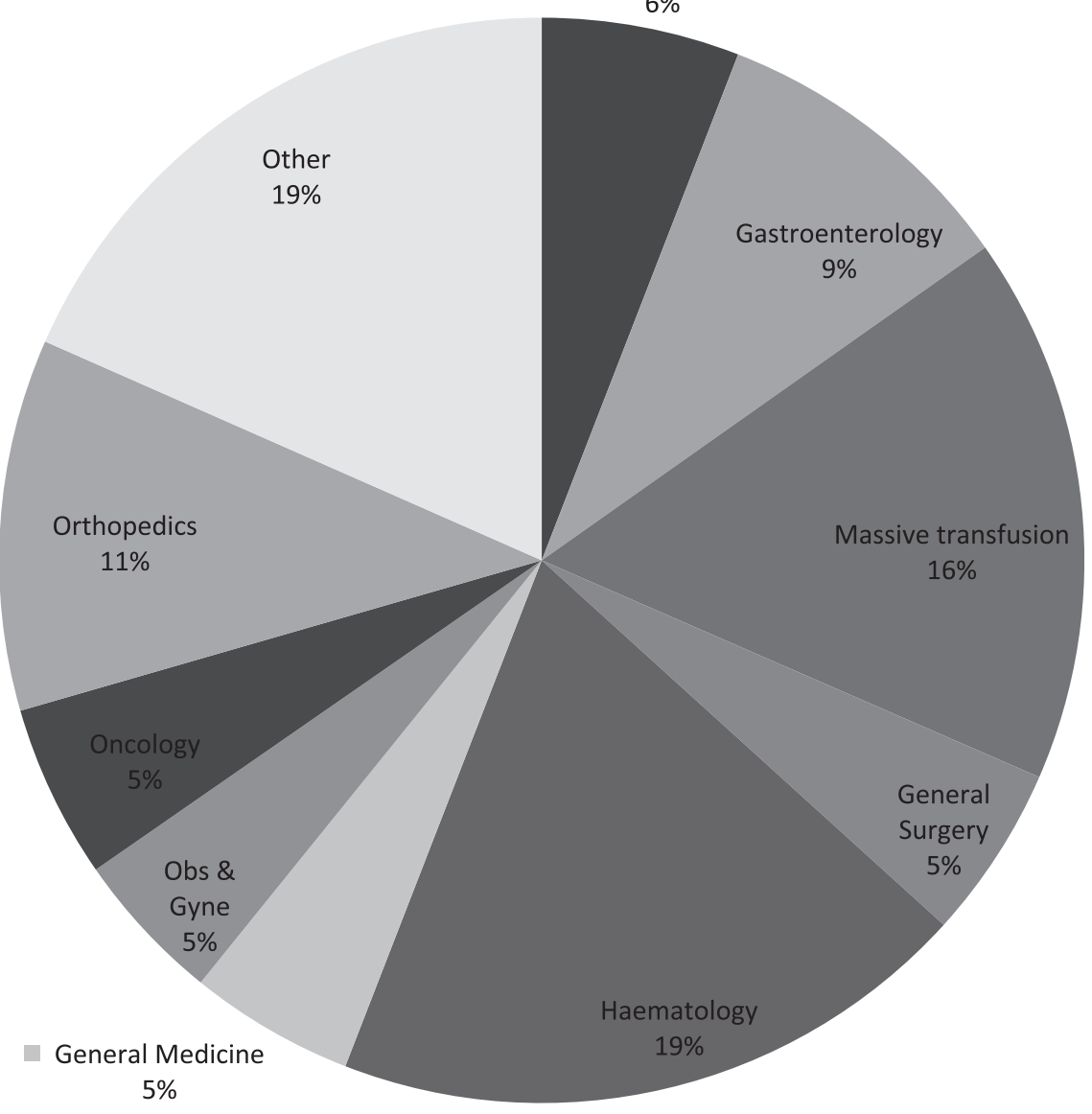

Fig. 1. Red blood cell utilisation by clinical department. 


\subsection{Targeting surgical procedures}

Cancelling elective surgical procedures reduces transfusions, but the majority of elective surgical procedures do not require transfusion. We therefore reviewed the Maximum Blood Order Schedule (MBOS) recommendations for transfusions to elective surgical patients to investigate whether it could be used to refine elective surgical deferral policy. The MBOS aims to minimise unnecessary crossmatching pre-operatively by listing the maximum number of red cell units that should be requested for common procedures. Only $0.3 \%$ of transfusions were for surgery where group and screen was not considered necessary, and a further $0.5 \%$ for surgery where a group and screen, but not a cross-match was deemed appropriate by the MBOS. Cancelling elective surgery where blood use is not anticipated will therefore have minimal benefit in conserving red cells during a shortage.

\section{Discussion}

Implementation of blood contingency plans requires local measures to reduce blood use in an efficient and equitable manner. National plans for blood shortages offer guidance for demand control strategies $[5,6]$, but implementation requires an understanding of which strategies are likely to be effective locally. The ideal strategy will minimise transfusion with the smallest impact on patient safety and wellbeing, be easy to implement and preferably not impact on procedures where transfusion is considered unlikely. Cancellation of elective surgical procedures, reducing transfusion triggers, implementation of guidelines, diverting patients to alternate hospitals and utilisation of frozen inventories have all been suggested as possible mechanisms to reduce demand, yet the relative efficacy of these strategies is uncertain $[1,3,10]$.

Using retrospective data, we found the greatest potential reduction in blood utilisation was to prevent inappropriate transfusions. This could reduce utilisation by as much as $17 \%$ with appropriate interventions to prevent inappropriate transfusions. There are a variety of methods for achieving this, with good results obtained from vetting all transfusions against accepted criteria prior to issue [11]. By definition, inappropriate transfusions produce minimal or no clinical benefit, so reducing then should be expected to have no adverse clinical outcome, and may even minimise the adverse effects of inappropriate transfusions. These may include minor reactions, anaphylactic, infectious and immune mediated events. While the incidence of major reactions is low there is considerable concern about the effects of transfusion on patient outcomes and any adverse event is preventable if the transfusion is not indicated. Inappropriate transfusions are not unique to the hospitals in this study, as rates such as this, or higher, have been reported by others across many institutions [12]. Therefore unless an institution has mechanisms in place to prevent inappropriate transfusions, it is likely that vetting is the preferred strategy to reduce demand during red cell shortages.

A concern regarding vetting of transfusions is that it may be considered rationing of an essential community resource. Rationing has been defined as "mechanisms that allow people to go without beneficial services," [13] but transfusions falling outside established guidelines are unlikely to benefit the recipients, and as such we don't believe this strategy is rationing. Restricting surgical procedures, even if elective however, is a form of rationing. Our recommended approach ensures the most appropriate use of resources, especially warranted given the ethics associated with blood donation, and in fact represents ideal practice. There are significant resource implications in adopting such a strategy, which accounts for the lack of widespread implementation of such a strategy [14]. Hospitals will need to consider the additional time required by transfusion medicine staff when formulating emergency management response plans for blood shortages. However, this may not always be adequate to cope with supply limitations, and not always necessary if there is accompanying reduction in demand.

Cancellation of elective surgery as an alternative strategy could decrease demand for blood by $9 \%$ if deferrals are restricted to only those patients not likely to suffer significant morbidity due to the delay. This is similar to $9.8 \%$ use in non-urgent medical and elective surgical indications found by Shorrt and co-workers [15]. That study was prospective, and had a high response rate over a broad range of indications, but the assessment of urgency was undertaken by laboratory personnel, who may not have questioned the urgency indicated by clinicians. The superiority of vetting transfusions over cancellation of surgery has also been demonstrated by Joseph and colleagues [16]. This study did not assess intra-operative transfusions and did not include a dedicated haemato-oncology unit. They suggested a saving of at least $23 \%$ of red cells by using more restrictive transfusion triggers, whereas only $11 \%$ could be saved by cancelling elective surgery.

If elective surgery deferral is adopted as a strategy only after eliminating inappropriate transfusions, it could reduce red cell usage by a further 5.5\%. Further reductions could be achieved by cancelling elective surgery only if there is assessment that the situation warrants an increased the risk to patients. Although not ideal, cancelling all except category 1 patients could reduce transfusions by a further $4.2 \%$. We recommend deferral of surgery only when vetting of transfusion has been inadequate, for clinical and ethical reasons. Some have argued that forcing of cancellation of surgery may also harm relationship between the blood bank and clinicians [1].

Unlike vetting, surgical deferral is a form of rationing. Deferring surgical procedures is a common strategy to deal with increased demand for operating theatre time or hospital bed-days, which may increase its acceptability, although it remains politically unpopular [1]. As many surgical procedures have a low likelihood of transfusion we found the MBOS useful to select surgical procedures that seldom use blood. It may therefore be feasible to select out procedures with a low risk of transfusion, using the MBOS as a guide, during times of shortage. Whether this is feasible may also depend on the cause for the supply and demand mismatch. There may be occasions, for example in response to a pandemic, when cancelling elective surgery may be required to free up other health care resources, and in this circumstance, the reduction in surgical blood use may prevent the 
need for additional measures - and it would be unwise to commit additional resources to vetting at this time if it is unnecessary if they could be deployed elsewhere. Understanding the potential benefit for surgical deferral is therefore useful, even if this is not to be the recommended first response to blood shortage.

We found significant differences in the potential for the hospitals to reduce demand, in part based on differing case mix. By corollary, similar strategies implemented in different institutions will have different impacts on the hospitals' functions. In order to ensure equity, and prevent the movement of patients cancelled from one institution to another, implementation of demand control measures should be consistent across regions when dealing with blood for transfusion, which is generally perceived as a community resource.

The study does have limitations, in particular as it was retrospective study on data previously collected data with the primary aim of assessing appropriateness and adverse reactions to transfusion. Clinical details for assessing the urgency of indications may have been incomplete as this was not the aim of the primary data collection. However, the major clinical details, including major diagnoses, procedures, bleeding and laboratory values for each case were collected. The similarity of our results for non-urgent transfusions to those of Shorrt and co-workers [15] adds to the validity. The decision to transfuse is often complex and the factors contributing to the decision may not have been fully recorded, and this may impact on the determination of whether a particular transfusion was appropriate. We attempted to overcome this by classifying transfusions as appropriate or inappropriate, by having two assessors look at the data independently, arriving at consensus where there was disagreement and by classifying transfusions as "possibly appropriate" where we felt there remained uncertainty. As a result, the study is likely to have a conservative estimate of the number of inappropriate transfusions and therefore the potential benefit from a strategy targeting these may be underestimated.

There are a number of potential strategies that we did not assess. Fresh blood products in Australia are derived from a single source, so there is no opportunity to diversify suppliers as others may [1]. Frozen red cells are kept by the supplier, but only for special circumstances such as rare blood groups, so this is not an option to deal with blood shortages at present. Others however have found this useful [3]. Attention by clinical staff to meticulous haemostasis, cell salvage and transfusion alternatives are also not assessed in this study. The strategy of reviewing all requests by a transfusion medicine specialist may identify such opportunities.

The study also did not determine for how long transfusion could be deferred. In particular, we could not determine whether inappropriate transfusions could be deferred permanently or whether patients would have subsequently required transfusion. These features of the study could lead to over-estimation of the potential benefit of postponing inappropriate transfusions as a demand control strategy when blood shortages exist for longer periods. Galloway and colleagues modelled the effect of a longer term ( 3 weeks) blood shortage and found it increasingly difficult the longer the shortage lasted as patients on short term deferral subsequently required transfusion [10]. Optimally, our results should be used to guide initial strategies, then, as the nature and duration of potential shortages become apparent, a search for longer term strategies, such as the use of erythropoietin or acquisition of further blood conservation equipment as needed by individual institutions.

Depending on the nature of the underlying cause, increases or decreases in blood transfusion demand may occur during periods of shortage. The duration and severity of supply shortage will also be variable. Therefore it is important to be aware of the options for transfusion demand control, how they may be implemented and the implications of each strategy. Our results suggest that optimising clinical transfusion practice, thereby minimising inappropriate transfusions, can enable significant reductions in blood utilisation. We suggest in the event of a red blood cell shortage that transfusion medicine specialists reviewing each transfusion request is the preferred initial strategy if demand remains constant.

\section{Conflict of interest}

None declared.

\section{References}

[1] McCarthy LJ. How do i manage a blood shortage in a transfusion service? Transfusion 2007;47:760-2.

[2] Gilcher RO, McCoombs S. Seasonal blood shortages can be eliminated. Curr Opin Hematol 2005;12:503-8.

[3] Erikson ML, Champion MH, Klien R, et al. Management of blood shortages in a tertiary care academic medical center: the Yale New Haven Hospital frozen blood reserve. Transfusion 2008;28:2252-63.

[4] Zimrin $A B$, Hess JR. Planning for pandemic influenza: effect of a pandemic on the supply and demand for blood products in the United States. Transfusion 2007;47:1071-9.

[5] Chief Medical Officer's National Blood Transfusion Committee. Development of an integrated blood shortage plan for the National Blood Service and hospitals. National Blood Service, London; 2004.

[6] National Blood Authority. National Blood Supply Contingency Plan Version 1, National Blood Authority, Canberra; 2008.

[7] Crispin PJ, Crowe BJ, McDonald AM. Blood Transfusion in the ACT: an insight into clinical decision making. Aust Health Rev 2005;29(2):240-5.

[8] National Health and Medical Research Council and Australasian Society of Blood Transfusion. Clinical Practice Guidelines on the use of blood components. National Health and Medical Research Council, Canberra; 2001.

[9] National Health and Medical Research Council. When does quality assurance in health care require independent ethical review? National Health and Medical Research Council, Canberra; 2003.

[10] Galloway MJ, Jane G, Sudlow J, Trattles J, Watson J. A tabletop exercise to assess a hospital emergency blood management contingency plan in a simulated acute blood shortage. Transfus Med 2008;18:302-7.

[11] Tuckfield A, Haeusler MN, Grigg AP, Metz J. Reduction in inappropriate use of blood products by prospective monitoring of blood transfusion request forms. Med J Aust 1997;167:473-6.

[12] Hebert PC, Schweitzer I, Calder L, Blajchman A, Giulivi A. Review of the clinical practice literature on allogeneic red blood cell transfusion. Can Med Assoc J 1997;156(Suppl. II):S9-S26.

[13] Hurst SA, Danis M. A framework for rationing by clinical judgement. Kennedy Inst Ethics J 2007;17:247-66.

[14] Tobin SN, Campbell DA, Boyce NW. Durability of response to a targeted intervention to modify clinician transfusion practices in a major teaching hospital. Med J Aust 2001;174:445-8.

[15] Shorrt J, Polizzitto MN, Waters N, et al. Assessment of the urgency and deferability of transfusion to inform emergency blood planning and triage: the Bloodhound prospective audit of red blood cell use. Transfusion 2009;49:2296-303.

[16] Joseph BG, Hendry C, Walsh TS. Red blood cell use outside the operating theatre: a prospective observational study with modelling of potential blood conservation during severe blood shortages. Transfusion 2009;49:2060-9. 